\title{
Traversing and sculpting scape through pattern development: Blending the natural and the built to promote ambiance in early architectural education
}

\author{
Kristin Barry ${ }^{1, *}$ and Sean Burns ${ }^{1}$ \\ ${ }^{1}$ Ball State University, College of Architecture and Planning, Department of Architecture, $2000 \mathrm{~W}$ \\ University Ave, Muncie, Indiana, USA
}

\begin{abstract}
In the formative years of their education, beginning design students are often equipped with several predetermined biases and stereotypical notions about architecture, with specific design intentions centered around how architecture should look, rather than how it should perform. The intent of this paper is to dispel these preconceptions by addressing the iterative design process through conceptual parameters of pattern and module creation, population, and manipulation in an effort to provide students with an alternative perspective for how architecture can create a dialogue with its environment and facilitate the transition from "thing seeing" to "pattern seeing." In two projects examined in this paper, students were asked to initiate the design process by exploring and testing the inherent strengths and constraints of pattern formations through the lens of a scape and constructed assembly, each of which would focus on principles of ambiance and spatial qualities, rather than relying on traditional architectural typologies. By developing these conceptual projects, students were able to break away from their preconceptions of what architecture is, and instead focus on what architecture could be with a reinvention of the design process.
\end{abstract}

\section{Introduction}

Acknowledging, employing, and manipulating patterns allow beginner design students the opportunity to give order to a system and test its constraints and capabilities, as influenced by the behaviors of constituent parts, to produce a performance within a composite assembly. Patterns have a measure of logic and rhythmic predictably due to the use of similar or modified elements arranged in an anticipated manner. There is also a degree of pliability within patterns, as inherent strengths and flexibilities allow them to differentiate, respond, and adapt to introduced criteria, demands, and desires, while remaining compositionally and systematically cohesive. Discovering all limitable bound conditions within pattern

* Corresponding author: kmbarry@bsu.edu 
configurations and alterations can encourage beginning design students to focus their attention on the realized parts-to-whole relationships towards appreciating how things behave and respond to one another internally, rather than championing the external appearance of their creations as they begin to create various ambiance settings within environmental design.

\section{Pattern as order, function, and design}

As designer and theorist Gyrogy Kepes introduces his essay Thing, Structure, Pattern, Process: "By deepest instinct, we reject chaos and try to order our experience. We seize upon means to bring coherence into the welter of signals furnished by nature as though our life depended on it--as it does" [1]. Kepes' ideas propose that inherently, beginner designers understand the world through known signs, symbols, and typologies, and it is only when they break from this preconception that they can fully understand and effectively communicate design. Paul Anderson and David Salomon expand upon this principle by introducing pattern as a way to deconstruct preconceptions and transition to the examination and construction of an unknown. They suggest that patterns can be multi-functional in their inherent capabilities and break from a canonical trope of architectural design, asserting that "Instead of form following function, patterns produce performances" [2]. Anderson and Salomon emphasize the performance or use of the object as that which matters most, as patterns do not simply relay identity, but become an active agent in the design process. The dichotomy of an object's use and its perception therefore is an instigator in the design, and consequently aesthetic qualities become the result rather than the driver.

Using the theories presented by Kepes, Anderson, and Salomon, the examination of understood or preconceived context can result in the deconstruction of known entities into fragments or patterns, and can encourage the reconstruction of these elements into a built environment within a new and innovative context. Deconstructing this environment into two elements, "constructed assembly" and "scape," helps to break down known typologies for beginning design students and focus on working within patterns, which can then purposefully interact to develop a contextual ambiance. As Kepes suggests, this allows students to move beyond "thing seeing" and toward "pattern seeing" [3]. Analyzing patterns, through the means by which the constituent elements are populated, deployed, and encouraged to advantageously interact, helps students identify inherent fundamentals of design such as connection and order, which can then be used in operational developments.

\section{Pattern seeing as ambiance}

To facilitate the initiation of new design iteration through more conceptual strategies, two projects given to beginner architecture students were developed to help students deconstruct their preconceived "thing seeing" and conversely transition to "pattern seeing" as a way to conceptualize ambient space. Each project required the design of a constructed assembly and accompanying scape through modules or patterns that would sensitively interact for a total user experience. Through an analysis of constraints and opportunities, students were encouraged to give the pattern, or module, agency in how it behaves, reacts, or responds to given criteria or the designer's intentions. Ambiance, therefore, is found in the understanding and purposeful sculpting of the scapes through the implementation of lighting, passage or movement, and exposure or enclosure strategies. As part of the design process, ambiance questions the interactions of these paired contexts and is driven by the way that the module or pattern responds, and not by an imposed or preconceived aesthetic ideal. 
Addressing design from the perspective of a module is one method of providing constraints and breaking down preconceptions for students to work within when initializing architectural design. In a project titled 'Part to Whole,' students were asked first to develop two disparate three-dimensional modules, or tessellations, and analyze them for constraints and possibilities with regard to flexibility, connection, and strength. Developing the modules as an individual action outside of a greater architectural design process encouraged students to think about the module as an individual actionable element with its own design criteria before thinking about its application as part of a total design. Students were not given any instruction on what the modules would later be used for, but instead were encouraged to think about the development of the small, repeatable object for its own outlined qualities, resulting in modules that responded to the characteristics given. As this project occurred early in the semester, students struggled with abstracting their ideas about the module from greater architectural thinking, and had difficulty focusing on the modules as individual elements and not as part of a greater scheme. Despite the hesitation felt, this ensured that students were not influenced by how the module would eventually be used and instead forced an individual design process centered on the element.

Following a critique of the modules with regard to flexibility, connection, and strength, students were asked to adapt the modules and then multiply them to be utilized in an ambient design where one module became an architectural constructed assembly and the other became an accompanying scape. While the modules were able to be adapted to better react based on the critique, they had to maintain the "spirit" and general aesthetic of the original modules to ensure that the students did not develop totally new designs based on the new requirements given, which would have negated the secrecy in the first part of the project. While difficult for students, blindly developing the module first was integral, to teach the students to design within restrictions and adapt based on critique. This ensured that the approach was on the part-to-whole development based on the reaction of the module and not on aesthetic intentions from the outset.

The second portion of the project required students to create a structure/enclosure/building and accompany scape using the adapted modules as the new constraint, and to think about the inherent qualities of the modules and their impact on space, with regard to ambiance. The new design process asked students to conceptualize ambiance through the purposeful application of lighting strategies, scale, and, enclosure, all of which should be facilitated by the design of the adapted modules. If the modules themselves were too enclosed and did not allow for light permeation individually, then spacing them out in the design to allow natural light through the offset of multiple modules was required. This presented a way of encouraging pattern seeing within the new building as a whole, suggesting to students that by creating multiple patterns with the modules that they could achieve an ambient space through the characteristics given of light, scale, and enclosure in different ways.

As a strategy to avoid common replication of patterns and encourage the adaptation of ambiance in both the structure and scape, students were asked to establish their own scale and program, and ensure that the building and scape had a sympathetic relationship. Rather than fall back on the common beginning design student preconception that buildings and landscapes exist independently and minimally react to each other, the project required that the structure and scape influence each other in multiple ways and to be designed to accommodate each other, suggesting that the ambient qualities of one could be carried into the design of the other. To reinforce this, both the scape and the architecture were viewed as 
permeable, emphasizing a three-dimensional relationship that would enhance experience, ambiance, and program.

The resulting designs exhibited a variety of strategies in the creation of ambiance through the modules, with some students choosing to identify a recognizable architectural program and work toward the creation of ambiance based on precedent, and others developing the building for ambiance unrelated to an established typology and instead focused specifically on the opportunities provided by the developed modules. Ultimately, the projects that used the modules as the design inspiration were more successful in creating ambiance qualities in the building or scape. The projects that relied on an existing building typology struggled overall to break away from preconceived notions about architectural design, as emphasized in the two parts of the project being assigned independently.

Student Jesse Lindenfeld's project [Fig. 1] embraced the idea that the landscape and building could both be structural and the design facilitated a difference in lighting and spatial qualities by embedding portions of the building within the permeable scape. The resulting design was strategic in its programmatic development of a library and maker-space, where the ambient qualities of the library required natural light, but the maker-space was embedded into a faceted landscape and purposefully fractured into different interacting programs. The design encouraged patrons to move between enclosed and open spaces, light and dark spaces, and into and out of the landscape as the program dictated, providing multiple ambient opportunities for exploration. The spaces were created through a three-dimensional module that was repeated and scaled to produce the building element and a folded module to create the landscape, which was flexible. Jesse's impression of the constraints was similar to many of the other students. He noted: "The constraint that provided the most challenge within this project was that of flexibility. The modules created had to be flexible enough to orient in different ways but also be manufacturable and sturdy. The way that I resolved this issue was starting from basic geometry, duplicating and folding from a base equilateral triangle. This repetition at a non-orthogonal angle allowed for easy reproducibility and interesting formal qualities" [4].

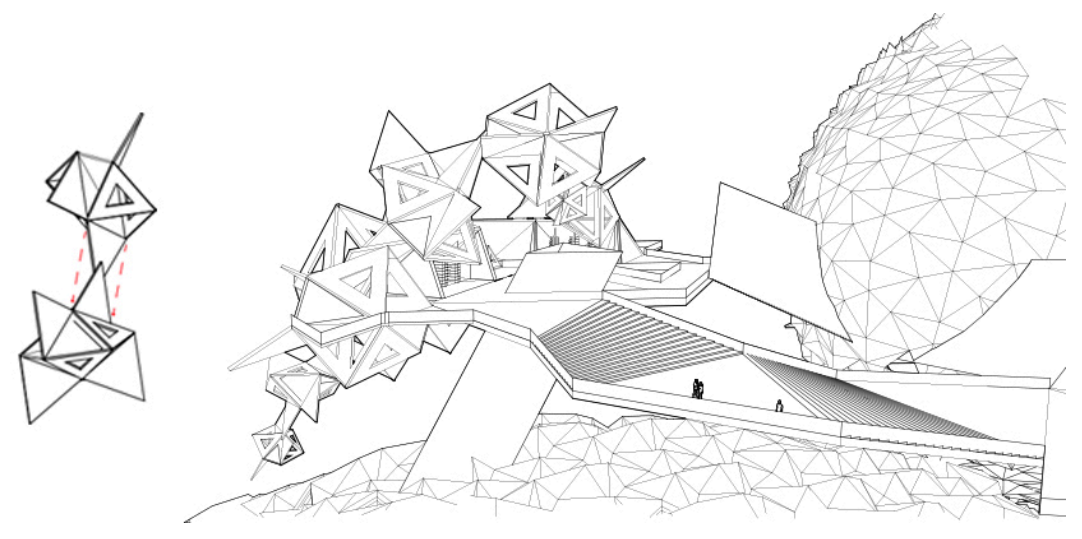

Fig. 1. One developed module and the corresponding building, incorporating a folded scape and constructed modular assembly as landscape and building for project 'Part to Whole'. Project and images by student Jesse Lindenfeld. 
Student Darrin Shedrow developed one of the more complex but adaptable modules for his building, which allowed for substantial flexibility in the repeated patterning that eventually made up the structure. By manipulating the connections between the modules, Darrin was able to open up the building to light in different ways and design a curvilinear structure that was impacted by site wind conditions. The landscape module, however, was much simpler and did not allow for the same flexibility in the design, resulting in less interaction between the landscape and building than the other two projects [Fig. 2]. All used ambiance and feeling within the space as inspiration behind the design instead of relying on building typology to initiate design, resulting in projects that were unique and creative in their approach for the first architectural project completed in their educational career.
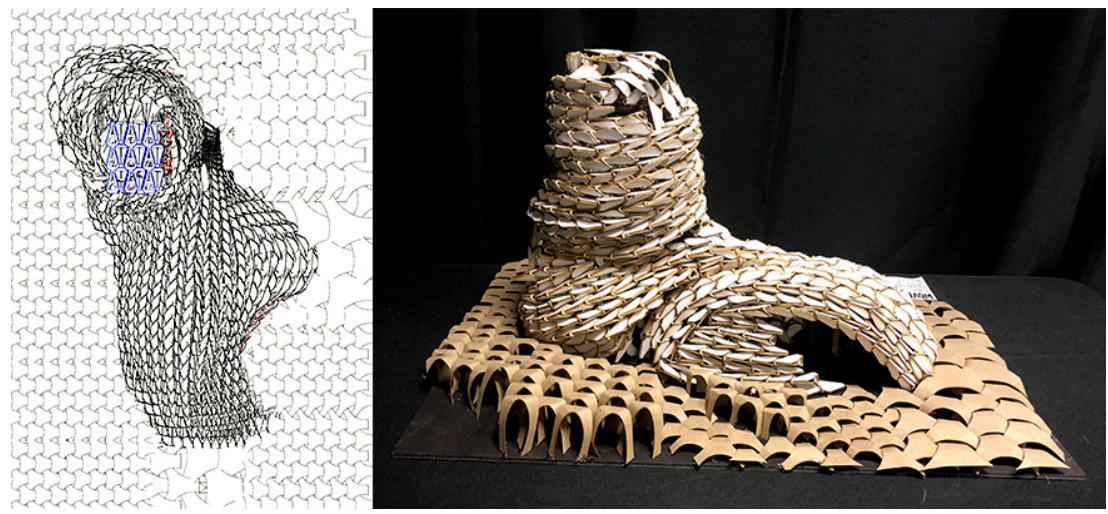

Fig. 2. Project module and diagram showing the flexibility of the constructed assembly module and scaling of the scape module to adapt to spatial requirements for project 'Part to Whole'. Project and images by student Darrin Shedrow.

\section{Performing patterns towards creating a contextual ambiance}

As with developing an adaptable module, folding is a procedure that demonstrates intention and consequence within the manipulation of an uncompromised membrane. The act of folding is indicative of creating or interrupting established pattern formations to achieve a desired response for a surface. A project entitled 'Pliant, [Dis]Engaged, Captured' asked firstyear environmental design students to blur the concepts of scape and constructed assembly by studying the performance capacities and compositional organizations of pattern formations as a means to create an ambiance within an existing underappreciated site. By considering folding, with its inherent consequences of seams, triangulated creases, pleats, and divisions, students created or manipulated pattern configurations as a procedure to capture and sculpt spaces with coveted programmatic intentions at a 1:1 scale. A separate structural framework system, which was responsive but disengaged from the folded skin at various moments, was implemented for the project. The structural framework and folded skin were required to sympathetically acknowledge and join one another in a manner that achieved a symbiotic relationship between the skin and bones within their project. As a result, folding became a vehicle to entice students to acknowledge the responsive behaviors within pattern formations, allowing the students to discover ways in which manipulated pattern formations might mediate light infiltration, capture and sculpt spaces, and suggest a new purpose for a site through creating ambient settings within their projects.

Working in teams, students began by studying the work of geometrist Ron Resch [5] to gain a better understanding of how folded tessellation systems allow paper to become both 
structural and flexible, as dependent upon the arrangement of various pattern formations and repetitive seams. Student teams translated their findings through iterations of folding operations to create a series of surfaces that explored the conditions of seams, triangulated creases, pleats, and divisions of a paper as informative elements to the overall surface membrane of a single sheet of paper. Additional sheets of paper were able to be included, only if attached along the full length of a creased edge, to examine how various strategies of combining multiple pattern formations might offer potentials for enclosure if imagined at a larger scale. As a final step, the students were asked to develop a framework system of bones, that would respond to their folded paper skin model by carrying forth its inherent geometric logic beyond the created surface as a means to provide additional structural support strategies for consideration within their designs [Fig. 3].

Upon completion of their preliminary studies, the student teams selected a site that was currently deemed uninformative in its programmed intentions and underutilized as a defined space within the interior confines of the College of Architecture and Planning at Ball State University. After observing the sites, the teams were tasked with activating the spaces by creating a 1:1 scale installation comprised of skin and bones to create a thematic ambiance using the folding operations and tectonic framework strategies learned in the previous explorations. So not to damage the existing conditions of the site, the students were not allowed to use any secondary means of connecting, bracing, or adhering their installation to the site beyond the direct contact of the framework or folded scape to the existing surroundings.
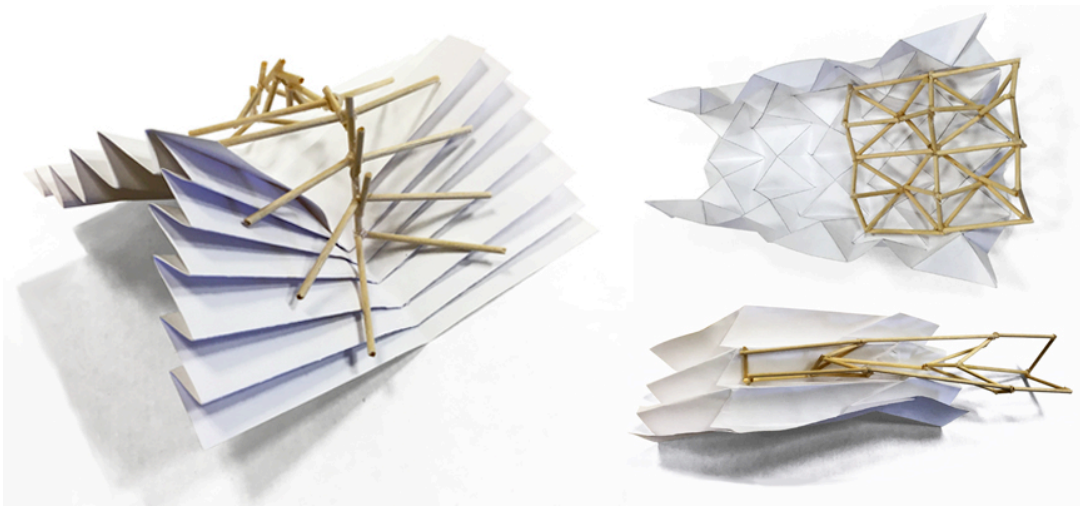

Fig. 3. Initial folded paper and tectonic structure studies by first-year environmental design students for 'Pliant, [Dis]Engaged, Captured' project.

First-year students Hao Xu and Nate Brower chose an interior site at a corner condition within a collaborate space of the building. The student team's initial analysis of the site determined that the space prohibited light intervention, was spatially undefined, and ineffective in promoting students' abilities to communicate and/or study. To address these concerns, Xu and Brower utilized the folded surface within their project to define and sculpt a space that encouraged peer interaction and discussions. The gesture of the skin was such that it followed the intended movement of the users as they traversed among the repurposed space. The formal gesture of the skin commenced by creating a portal overhead to invite users within the sculpted space. Upon entering the site, the folded membrane then descended to a establish a more private setting with an accompanying built-in seating area. Upon this moment of pause, the skin then ascended to lead the users out of the project and back through the created entry portal. The student team utilized a singular folded strategy for the geometric 
pattern formations of the skin and sympathetic bones, yet modulated and adapted the size of this pattern to best achieve the desired formal gesture along the path of travel for the project. Further, as the absence of light was noted as a flaw of the original environment of the site, the students integrated artificial lighting within the interior envelope of their folded skin and removed panels of their skin's undulating pattern at specific moments to reveal the interplay between the tectonic bones and the folded membrane [Fig. 4].

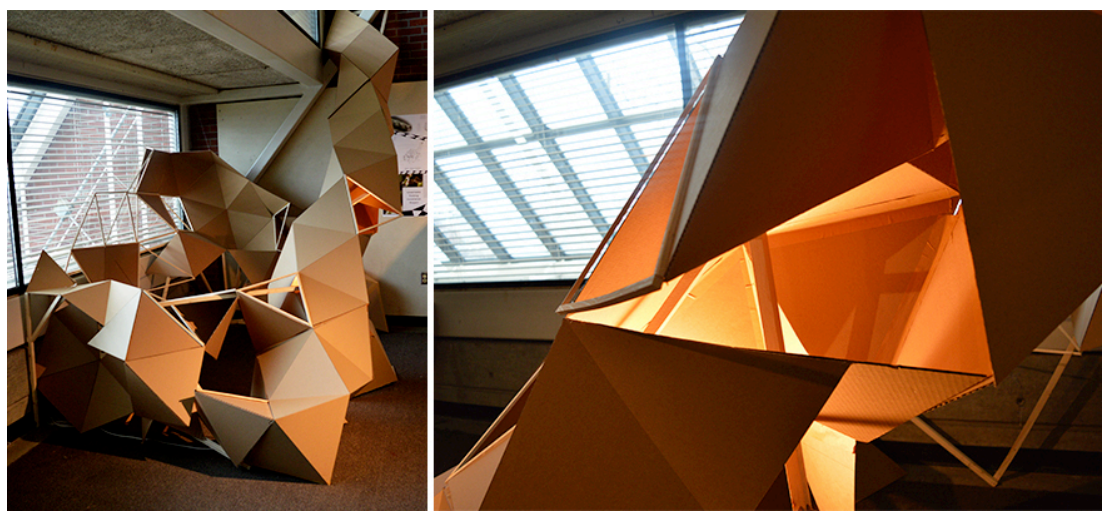

Fig. 4. Final installation design and fabrication and detail of symbiotic connection between skin and bones by first-year environmental design students Hao Xu and Nate Brower for 'Pliant, [Dis]Engaged, Captured' project.

The student team of Mia Baker and Kirsten Toole adopted a portion of the building's interior four-story tall atrium that was contiguous to the architectural library as their site for the project. Upon studying how people circulated and interacted within and around their designated space in its existing setting, the student team decided to focus on redefining the boundary conditions, in the $x-y-z$ axes, to establish a more intimate scale as their overarching strategy and repurpose their site as a place to read and contemplate, given its proximity to the library. To achieve this charge, the students studied several iterations of scaled study-models to strategize various means to soften the height of their site, as well as capture and define a personal space that was to be shielded from other activities within the atrium. In its final configuration, the pattern formation of the folded skin was predicated on its ability to pivot at specific creases to achieve the form of the newly-established sculpted demarcation. Ultimately, the students' design of the skin was such that it emerged from the ground, utilized the tectonic framework bones to brace itself to a low-height wall near an open staircase, and then reposition itself to create a canopy overheard of the implemented seating area. The formal gesture of the project was further influenced by the project team's desire to mitigate the noise of the atrium, protect the inhabitant of their site form the wind emanating from the front exterior doors of the atrium space, and deflect the abundance of outdoor light at the newly created seating area [Fig. 5]. 


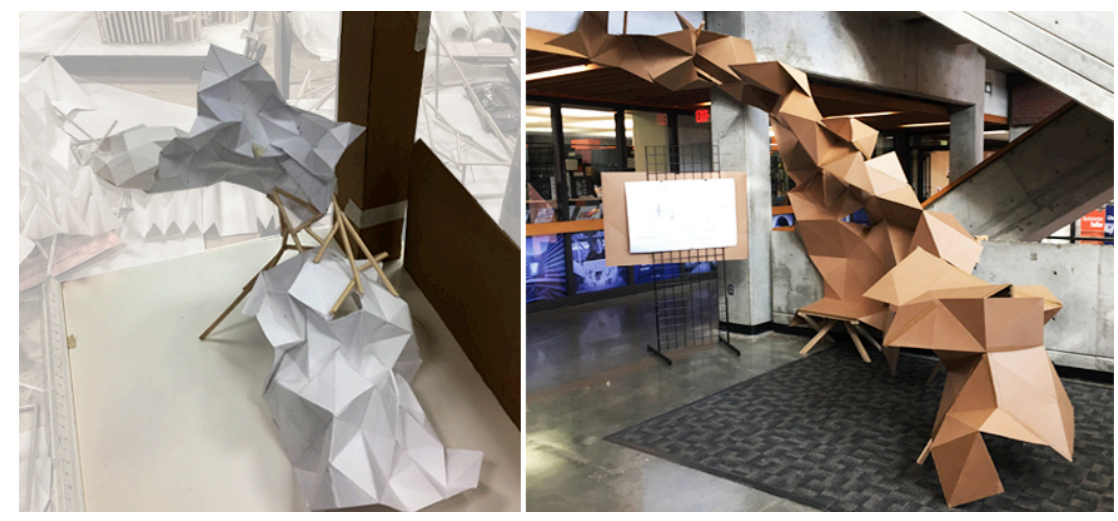

Fig. 5. Folded paper process study model and final installation design and fabrication by first-year environmental design students Mia Baker and Kirsten Toole for 'Pliant, [Dis]Engaged, Captured' project.As commented by student Mia Baker, "The project demonstrated that design does not always commence with trace paper, but instead, it can start with observing, listening and developing a design language. Our team used a process of making and collaborating, all new skills to us at the time, to help cultivate a specific ambiance based on our personal shared experience of our given site. I learned how to abstract and reinterpret structure and surface as both interconnected and interdependent systems within a compositional design for an intended environment. Further, as the project was at full scale, it allowed us to directly and physically create and refine our solution to encourage users to interact with the created scape" [6].

\section{Conclusions}

Each of these projects introduced principles of pattern manipulation towards conceptualizing architectural design as a marriage of elements associated with the ground and among the sky. Having students conceptualize ambiance through these principles is integral to their development as beginner architectural students, as it introduces inherent qualities or principles of design and their influence on the final product, focusing on the iterative process and emphasizing concept and reaction as developmental tools. Establishing parameters ensures that students apply rigorous analysis of behaviors in the creation of the scapes, rather than allow aesthetics to drive the process when designing ambient space.

\section{References}

1. G. Kepes, The New Landscape in Art and Science, 204 (1956)

2. P. Anderson, D. Salomon, The Architecture of Patterns, 33 (2010)

3. G. Kepes, The New Landscape in Art and Science, 204-207 (1956)

4. J. Lindenfeld, "Part to Whole." E-mail message to primary author, (December 3, 2018)

5. R. Resch, E. Armstrong, Paper and Stick Film, (1970)

6. M. Baker, "Pliant, Dis[Engaged], Captured Project." E-mail message to secondary author, (November 19, 2018) 